\title{
Accessible Region Conformation Capture (ARC-C) gives high-resolution insights into genome architecture and regulation
}

\author{
Ni Huang, ${ }^{1,2}$ Wei Qiang Seow, ${ }^{1,2}$ Alex Appert, ${ }^{1}$ Yan Dong, ${ }^{1}$ Przemyslaw Stempor, ${ }^{1}$ \\ and Julie Ahringer ${ }^{1}$ \\ ${ }^{1}$ The Gurdon Institute and Department of Genetics, University of Cambridge, Cambridge CB2 1QN, United Kingdom
}

\begin{abstract}
Nuclear organization and chromatin interactions are important for genome function, yet determining chromatin connections at high resolution remains a major challenge. To address this, we developed Accessible Region Conformation Capture (ARC-C), which profiles interactions between regulatory elements genome-wide without a capture step. Applied to Caenorhabditis elegans, ARC-C identifies approximately 15,000 significant interactions between regulatory elements at 500-bp resolution. Of 105 TFs or chromatin regulators tested, we find that the binding sites of 60 are enriched for interacting with each other, making them candidates for mediating interactions. These include cohesin and condensin II. Applying ARC$\mathrm{C}$ to a mutant of transcription factor BLMP-1 detected changes in interactions between its targets. ARC-C simultaneously profiles domain-level architecture, and we observe that $C$. elegans chromatin domains defined by either active or repressive modifications form topologically associating domains (TADs) that interact with A/B (active/inactive) compartment-like structure. Furthermore, we discover that inactive compartment interactions are dependent on H3K9 methylation. ARC-C is a powerful new tool to interrogate genome architecture and regulatory interactions at high resolution.
\end{abstract}

[Supplemental material is available for this article.]

The development and application of chromosome conformation capture methods have been instrumental in shaping our understanding of genome topology (Dekker et al. 2002; Sati and Cavalli 2017). The basic premise is that if two regions of the genome are in close proximity within the nucleus, they can be ligated together after the DNA is fragmented. The products generated by proximity ligation can then be determined using sequencing or PCR-based methods. A diverse array of " $\mathrm{C}$ " methods have been developed, and their use has revealed interactions between regulatory elements, self-interacting topologically associating domains (TADs), and a "compartment" structure of the genome in which regions of similar activity interact like with like (Dekker et al. 2013).

The Hi-C method enables genome-wide assay of chromatin interactions (Lieberman-Aiden et al. 2009). In most Hi-C-type methods, DNA is fragmented relatively uniformly using restriction or other enzymes for capture of interactions between all regions of the genome (Davies et al. 2017). These genome-wide Hi-C methods are powerful for mapping chromatin domain structures such as TADs, but because of low resolution at reasonably achievable sequencing depth, they are not able to profile interactions between individual promoters and enhancers (Davies et al. 2017). These instead are typically mapped by enriching a Hi-C library for regions of interest, such as collections of promoters or regions bound by transcription factors, using oligo-based capture methods, for example, Capture-C (Hughes et al. 2014), Capture Hi-C (Mifsud et al. 2015), or Targeted DNase Hi-C (Ma et al. 2015). This requires the user to choose and synthesize regions to target and limits the

\footnotetext{
${ }^{2}$ These authors contributed equally to this work. Corresponding author: j.ahringer@gurdon.cam.ac.uk

Article published online before print. Article, supplemental material, and publication date are at https://www.genome.org/cgi/doi/10.1101/gr.275669.121. Freely available online through the Genome Research Open Access option.
}

assay to specific subsets of elements, adding expense and complexity to the assay.

Chromatin at regulatory elements is known to be relatively accessible to nucleases, which has enabled their mapping using sensitivity to DNase I or Tn5 transposition (Tsompana and Buck 2014). A higher concentration of DNase I has been used to fragment chromatin uniformly in DNase-Hi-C and Targeted DNase Hi-C methods (Ma et al. 2015). We reasoned that using a low concentration of DNase I to bias cutting toward accessible chromatin would enrich a chromatin interaction library for interactions between regulatory elements and enable their profiling while still having sufficient information for interrogating larger-scale architecture. This would avoid limiting high-resolution information to user-defined regions (as in target capture methods) and allow mapping domain structure in the same experiment. Using this principle, we developed Accessible Region Conformation Capture (ARC-C).

\section{Results}

The steps of ARC-C are illustrated in Figure 1A (Methods). Nuclei fixed with formaldehyde are treated with a relatively low concentration of DNase I to bias for cutting at accessible chromatin and give maximal recovery of interactions at regulatory elements (Supplemental Fig. S1; Methods). Ends are then repaired and DNA ligated to join ends in close proximity. The library is then made in nucleus using Tn5 tagmentation (Buenrostro et al. 2013), amplified, size-selected for inserts $<400 \mathrm{bp}$, and pairedend sequenced. For data processing, we first identify "valid" high-quality uniquely mapping read pairs. We then define

(C) 2022 Huang et al. This article, published in Genome Research, is available under a Creative Commons License (Attribution 4.0 International), as described at http://creativecommons.org/licenses/by/4.0/. 


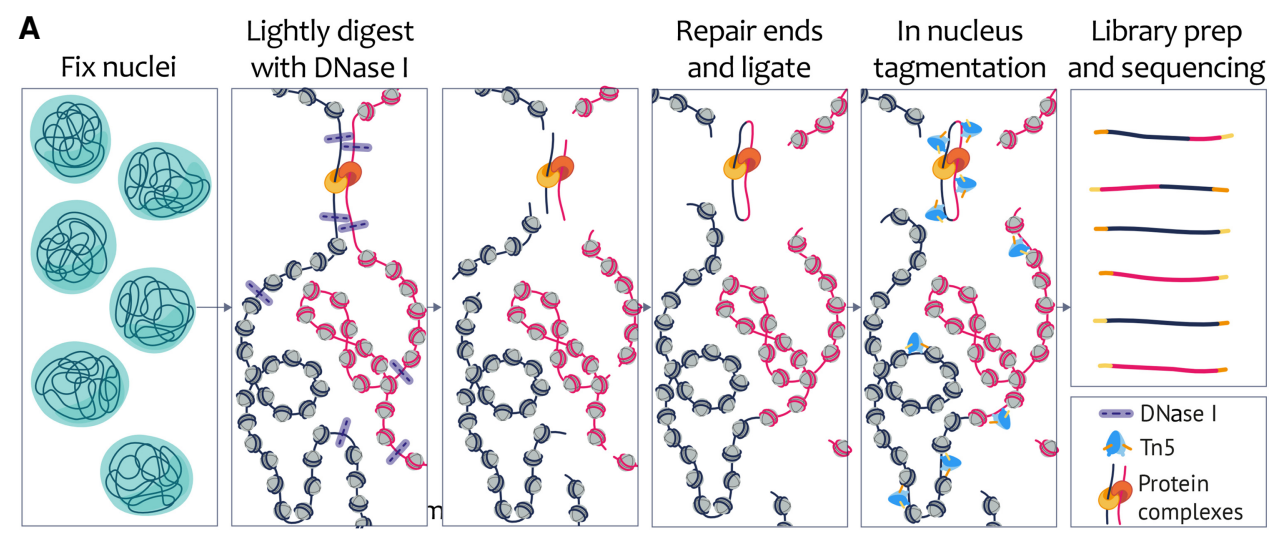

B Data processing

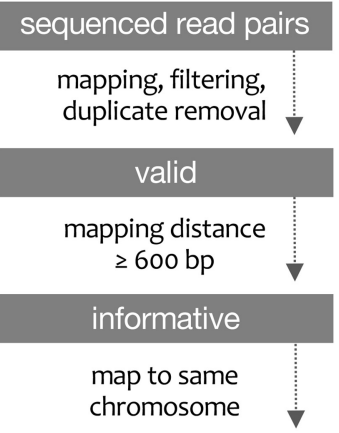

cis-informative

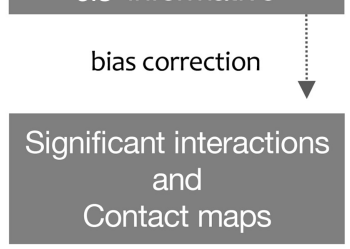

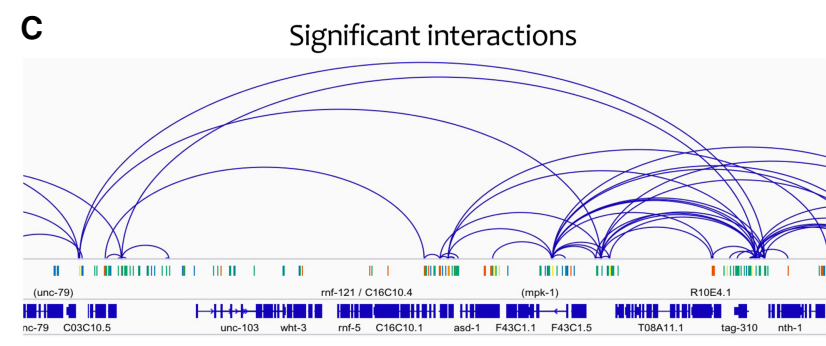

D X Chromosome contact maps

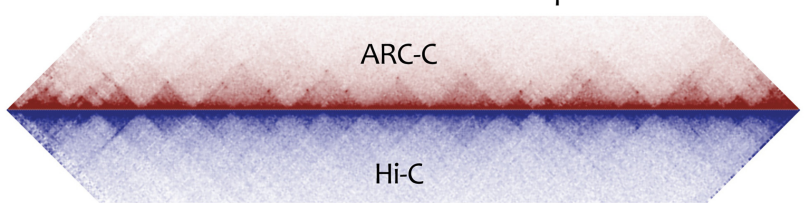

E Interaction strength between top 25 rex sites

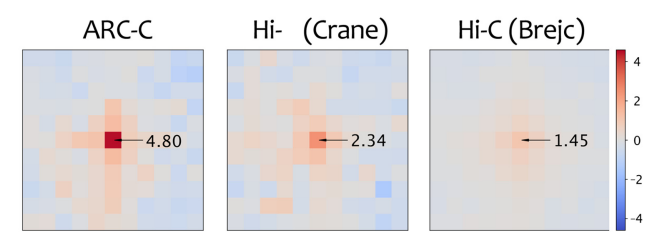

Figure 1. ARC-C method and comparison with Hi-C. (A) ARC-C cartoon. (B) Data processing steps. (C) Integrative Genomics Viewer (IGV) (Robinson et al. 2011) screen shot showing significant interactions in a $275-\mathrm{kb}$ window of $\mathrm{Chr}$ III $(4,075,000-4,300,822)$. Regulatory elements (protein-coding promoters, red; unassigned promoters, yellow; enhancers, green; unknown activity, blue) (Jänes et al. 2018) and genes are displayed below. (D) Comparison of ARC-C and Hi-C X Chromosome contact maps. Hi-C data are from Crane et al. (2015). (E) Aggregate contact analysis plots (Rao et al. 2014) showing signal between top 25 rex sites (Crane et al. 2015) at 10-kb resolution and a distance range of $100 \mathrm{~kb}$ to $4 \mathrm{Mb}$ for ARC-C (this study) and two C. elegans Hi-C maps (Crane et al. 2015; Brejc et al. 2017). Arrows indicate the linear enrichment of rex-rex interactions (at the central 10-kb square) relative to interactions between other regions. rex-rex interaction strength was statistically significant $(P<0.001$; permutation test, see Methods) in all three maps.

"informative" read pairs that have captured ligation events as those mapping $>600 \mathrm{bp}$ apart or to different chromosomes. Finally, we use "cis-informative" read pairs (those mapping to the same chromosome) to construct chromosome-wide contact maps and to call significant interactions following bias correction (Fig. 1B-D; Supplemental Fig. S2; Methods).

We applied ARC-C to Caenorhabditis elegans L3 chromatin, preparing libraries from three biological replicates. Data from all replicates were highly concordant (Supplemental Fig. S2). To increase the power to profile interactions, all cis-informative reads were pooled, resulting in 12 million read pairs for analysis (Supplemental Fig. S2). As expected, cis-informative read pairs were enriched at regulatory elements $-43.7 \%$ of reads overlap reg- ulatory elements (REs), which comprise $21.1 \%$ of the genomeand REs are from Jänes et al. (2018). Cis-informative read pairs are produced by ARC-C with an efficiency similar to those of capture methods (Supplemental Fig. S3).

Previous studies of C. elegans genome topology using Hi-C identified and characterized large roughly 200 -gene self-interacting domains on the $\mathrm{X}$ Chromosome regulated by the dosage compensation complex (DCC) (Crane et al. 2015; Brejc et al. 2017). Large domains were also observed on autosomes, although they were weaker, but smaller self-interacting TADs similar to those in Drosophila and vertebrate genomes, which typically contain one to several genes, were not detected (Dixon et al. 2012; Nora et al. 2012; Sexton et al. 2012; Dekker and Heard 2015; Brejc et al.

\section{Genome Research}

www.genome.org 
2017). The $X$ Chromosome domain boundaries were shown to be enriched for DCC binding at recruitment element on X (rex) sites, and enrichment for contacts between rex-containing regions was observed (Crane et al. 2015; Brejc et al. 2017).

We found that ARC-C recapitulated the $\mathrm{X}$ Chromosome domains and insulation profiles observed by $\mathrm{Hi}-\mathrm{C}$ and more sensitively detected interactions between rex sites (Fig. 1D,E; Supplemental Figs. S4, S5). ARC-C maps and insulation profiles from the autosomes are also highly similar to Hi-C maps including detection of the previously reported preferential interactions within the large central and two distal chromosome blocks of autosomes (Supplemental Figs. S4, S5; Crane et al. 2015). These results show that ARC-C can profile large-scale domain architecture and suggest that it can improve detection of specific interactions between regulatory elements.

We next investigated the potential formation of smaller TAD-like domains. Although these were not clearly visible in the contact matrix, we considered that genomic domains defined by chromatin modifications might form selfinteracting domains, because in other animals, marking patterns within individual TADs and small compartmental domains are relatively uniform (Sexton et al. 2012; Rowley et al. 2017). In C. elegans, histone modification domains segment most of the autosomal genome into active and H3K27me3 (aka "regulated") domains (Gaydos et al. 2012; Evans et al. 2016). Active domains are enriched for broadly and germline active genes marked by H3K36me3 and other modifications associated with gene activity, and the alternating $\mathrm{H} 3 \mathrm{~K} 27 \mathrm{me} 3$ domains cover genes that have regulated expression (Gaydos et al. 2012; Evans et al. 2016). Both domain types have a median gene number of three, with median lengths of $22 \mathrm{~kb}$ for active domains and $19 \mathrm{~kb}$ for H3K27me3 domains (Evans et al. 2016). The levels of valid ARC-C signals were only slightly higher in active domains compared to H3K27me3 domains, enabling its use to investigate whether the active and H3K27me3 chromatin domains are spatially separated and form TAD-like structures (Supplemental Fig. S6A,B).

To investigate whether the active and H3K27me3 chromatin domains are spatially separated and form TAD-like structures, we visualized and compared interaction frequencies in aggregate within active or H3K27me3 domains and their neighboring chromatin. Supporting the spatial separation of both domain types, we found enrichment for interactions within domains, visible as a central square of higher signal (Fig. 2A,E). To further test topological separation, we calculated average insulation scores for active and H3K27me3 domains, where low values indicate regions with low contact frequency (Crane et al. 2015). We observed local mini-

E

\section{Compartment assay: interactions between domains of same type}

B wild-type

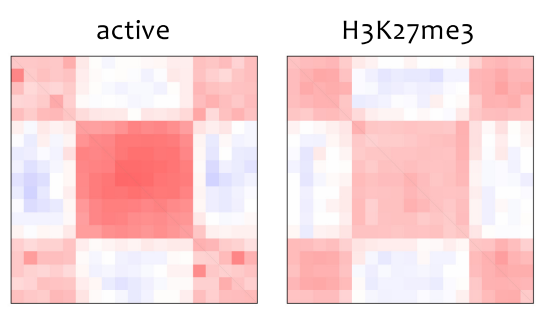

D

met-2 set-25

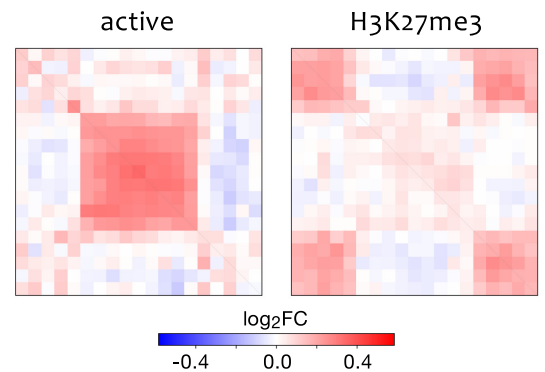

$\mathrm{H}_{3} \mathrm{~K} 27 \mathrm{me} 3$

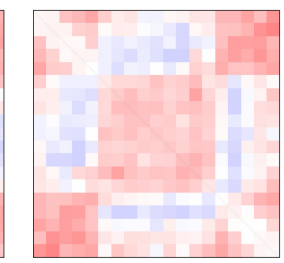

$\log _{2} \mathrm{FC}$

\begin{tabular}{|c|c|c|c|}
\multicolumn{2}{c|}{ Domains } & \multicolumn{2}{c|}{ Compartments } \\
\hline 2.4 & 5.7 & 23.0 & 9.9 \\
\hline 9.7 & 7.2 & 23.0 & 1.0 \\
\hline 1.3 & 1.3 & 1.0 & -9.9 \\
\hline
\end{tabular}

Figure 2. Chromatin modification domains form TADs that have compartment structure. $(A, C)$ Active and $\mathrm{H} 3 \mathrm{~K} 27 \mathrm{me} 3$ chromatin modification domains were aligned and contact map signal aggregated in withing regions and neighboring regions. Higher signal in the central square shows enrichment for in the domain interactions, indicative of TAD structure. $(B, D)$ All possible pairs of inter-domain contacts square shows that domains interact more frequently with domains of the same type than with domains of opposite types, indicative of compartment structure. $(E)$ Quantification of enrichment in contact frequencies (as percent differences) for domains and compartments in wild-type and met- 2 set-25 mutants. shown. For compartments, the percent difference in contact frequency between domains of the same type versus domains of different type is shown. See Methods for details.

ma of insulation profiles flanking active and H3K27me3 domains, supporting the existence of TAD boundaries (Supplemental Fig. S6C). These results indicate that active and H3K27me3 domains have TAD structure, because they are enriched for self-interactions and insulated. The compact nature of the C. elegans genome and the relative weakness of these domains compared to TADs in Drosophila and mammals may have prevented their detection from de novo analyses.

We next investigated whether domains of the same type (active or H3K27me3) interact with each other to form an A/B (active/inactive) compartment-like structure. For these analyses, we aggregated ARC-C signal either between active domains or between H3K27me3 domains. This revealed that domains show preferential interactions with those of the same type (Fig. 2B,E). Although weaker, domains and compartments detected using ARC-C are also apparent in similar analyses of Hi-C data (Supplemental Fig. S7B). We conclude that $C$. elegans active and H3K27me3 chromatin domains form TADs and that TADs of the same type interact with each other to form a compartment-like 
structure similar to A/B (active/inactive) compartments of other animals. We note that compartment strength appears to be higher than that of TAD strength. This may be related to the apparent lack of insulator proteins in C. elegans (e.g., Heger et al. 2009). A possibility is that compartment interactions between domains of like type contribute to the observed TAD structures.

The mechanisms of compartment formation are not well understood, and few factors that affect compartment structure are known. We considered that histone modifications may be important because they largely differ within active and inactive compartments (Lieberman-Aiden et al. 2009). H3K9 methylation is a good candidate because heterochromatic $\mathrm{H} 3 \mathrm{~K} 9 \mathrm{di}$ - and trimethylation is predominantly found in inactive compartments, and interactions within heterochromatin have been suggested to drive compartment structure (Falk et al. 2019).

The two distal regions of C. elegans autosomes contain most of the H3K9 methylation and associate with the nuclear lamina, whereas the central region contains little H3K9 methylation (Ikegami et al. 2010; Liu et al. 2011). Active and H3K27me3 domains alternate across both distal and central chromosomal regions, with H3K27me3 marking largely coinciding with H3K9me3 in the distal arm regions (Gerstein et al. 2010; Liu et al. 2011; Evans et al. 2016; Ahringer and Gasser 2018). H3K9me2 and H3K9me1 are also predominantly found in the distal arm regions, but are found in both active and H3K27me3 domains (Gerstein et al. 2010; Liu et al. 2011; Evans et al. 2016; Ahringer and Gasser 2018).

Nearly all H3K9 methylation in C. elegans is generated by two enzymes: MET-2 (a histone methyltransferase similar to human SETDB1) and SET-25 (Towbin et al. 2012). Mutants lacking both enzymes are viable and fertile but have undetectable H3K9 methylation and lose nuclear lamina association of chromatin normally marked by H3K9 methylation (Towbin et al. 2012), indicating a potential role of $\mathrm{H} 3 \mathrm{~K} 9$ methylation in large-scale nuclear organization.

To investigate whether H3K9 methylation plays a role in compartment structure, we performed ARC-C on met- 2 set- 25 double mutants. We observed that active and H3K27me3 chromatin domains formed TADs relatively normally, although there were small changes in interaction strength (Fig. 2C,E). Compartment interactions between active domains were also maintained (Fig. 2D,E). In contrast, the met-2 set-25 double mutants lacking H3K9 methylation showed a nearly 10-fold loss of compartment interaction strength between H3K27me3 domains (Fig. 2D,E). We conclude that H3K9 methylation is necessary for compartmentalization of H3K27me3 domains.

We wondered whether the requirement for H3K9 methylation was specific for $\mathrm{H} 3 \mathrm{~K} 27 \mathrm{me} 3$ domains that are normally H3K9me marked in wild type, or alternatively was needed for the compartment structure of both marked and unmarked domains. To investigate this, we separated domains into H3K9me marked and unmarked groups and then assayed compartment and TAD strength in wild-type and met-2 set-25 mutants. We found that loss of H3K9 methylation strongly reduced the compartment strength of H3K27me3 domains irrespective of whether the domains were marked by any methylated form of H3K9 in wild type (Supplemental Fig. S7C). These results indicate that H3K9 methylation, predominantly found on the distal arms, facilitates the compartment structure of H3K27me3 domains across the chromosomes, even in regions that lack H3K9 methylation. Given the known role of H3K9 methylation in tethering distal chromatin to the nuclear envelope (Towbin et al. 2012), we hypothesize that tethering of distal arm H3K27me3 domains may be important for the compartmental structure of H3K27me3 domains across the autosomal chromosomes.

A parallel studying using $\mathrm{Hi}-\mathrm{C}$ reported differences in interaction frequencies within the large distal and center autosomal regions in met-2 set-25 mutants compared to wild type, such as disruption of interactions within the central regions distal arms, which we confirmed using ARC-C (Supplemental Fig. S8; Bian et al. 2020).

Despite the strong effect on H3K27me3 compartment strength, the loss of H3K9 methylation did not have a widespread or specific effect on gene expression in H3K27me3 domains, because met-2 set-25 mutants displayed similar levels of gene expression dysregulation in active and H3K27me3 domains (Supplemental Fig. S9; Supplemental Table S1). Altered genes were predominantly up-regulated, and gene expression increases were associated with genes normally marked by H3K9 methylation irrespective of whether the gene was located in an active or H3K27me3 domain (Supplemental Fig. S9). We conclude that H3K9 methylation is an important mediator of inactive compartment structure, but that compartment structure itself has little effect on gene expression.

We next investigated the ability of ARC-C to profile interactions between regulatory elements. We previously defined 15,714 promoter and 19,231 enhancer elements in C. elegans (Jänes et al. 2018). To identify chromatin interactions between these and other genomic elements at high resolution, we separated the genome into 500-bp bins and identified those that interacted significantly with other bins, taking into account distance and coverage biases (Methods). This identified 14,992 chromatin interactions within a distance of $1 \mathrm{~kb}$ to $1 \mathrm{Mb}$ (Supplemental Table S2). The interactions involve 9733 different regions, 95\% of which overlap an annotated promoter or enhancer (Fig. 3A; Supplemental Table S3). Therefore, ARC-C has the power to map interactions between regulatory elements at 500-bp resolution.

Promoters are most prevalent among the significant interactions, accounting for $62 \%$ of interacting elements, and they are involved in $86 \%$ of the interactions (Fig. 3A). Half of the significant interactions are relatively short range (within $20 \mathrm{~kb}$ ), and in this size range we observed a similar number of P-P and P-E interactions (Fig. 3B). However, at longer distances, promoter-promoter interactions predominate. We found that genes connected by promoter-promoter interactions had correlated gene expression (Fig. 3C) and that the correlation is strongest for pairs with highly regulated expression, that is, those with high coefficients of variation of gene expression $(\mathrm{CV})$, suggesting that such genes are in proximity with each other when expressed.

To identify proteins that are candidates for mediating interactions in C. elegans, we screened for transcription factors and chromatin regulators for which binding sites show significantly enriched interactions in the ARC-C contact map (Methods). Of 105 proteins tested, 60 chromatin regulators or TFs had this property (Fig. 3D; Supplemental Figs. S10, S11; Supplemental Table S4). Similar assessment of two Hi-C maps (Crane et al. 2015; Brejc et al. 2017) identified only five proteins whose binding sites showed significantly enriched interactions; however, trends were similar to those observed using ARC-C (Supplemental Fig. S10). As expected from the role of the condensin I DC complex in mediating $\mathrm{X}$ Chromosome interactions between rex sites (Crane et al. 2015), the binding sites of condensin components were highly enriched for interactions (Fig. 3D). Other proteins of note are cohesin SCC-1 and loading factor PQM-85/NIPBL, consistent with similar enrichment in mammals and with the role of cohesin in loop

\section{Genome Research}

www.genome.org 
A

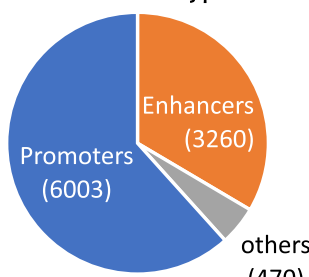

(470)

B

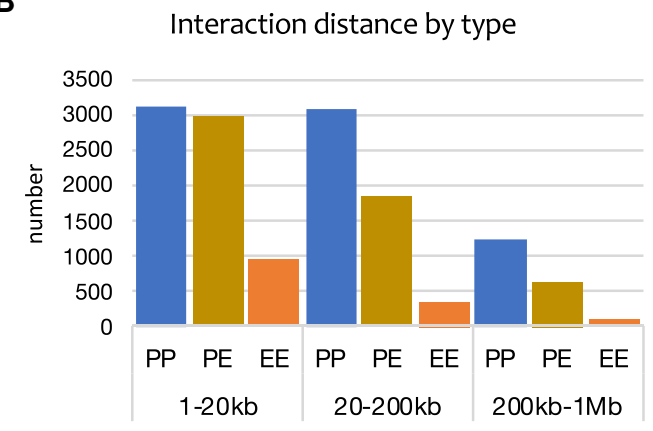

C

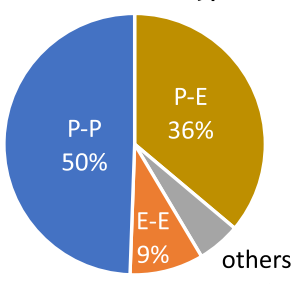

$5 \%$

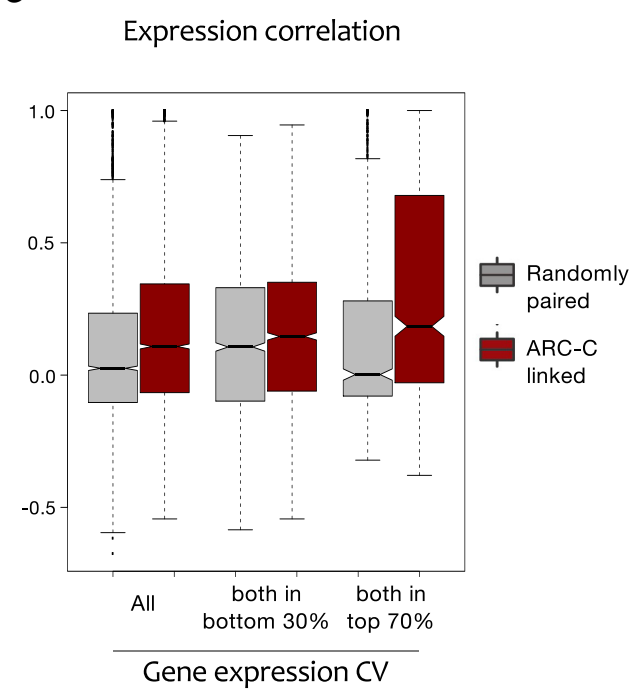

D
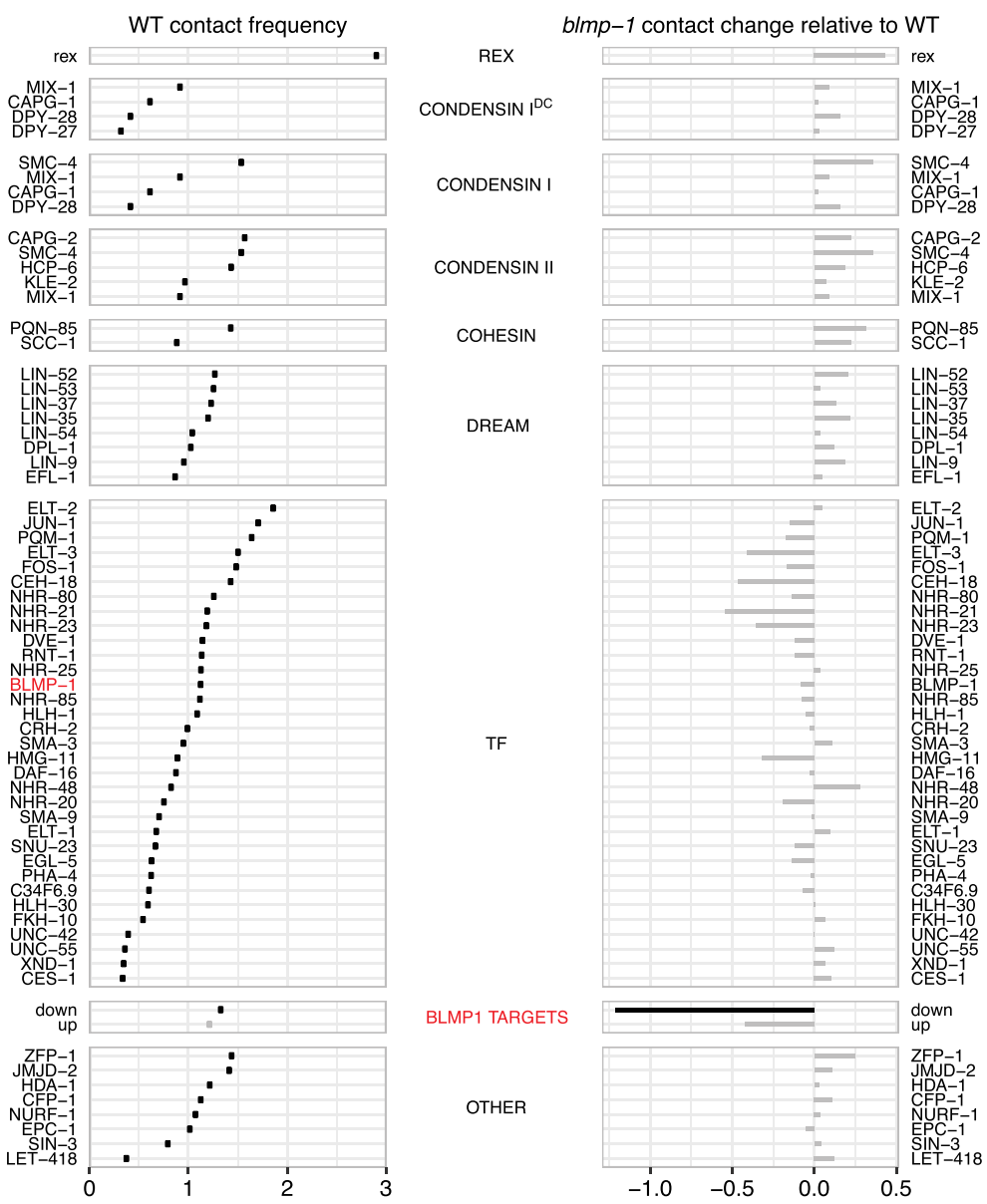

Increased contact frequency

Normalized difference

relative to background $\left(\log _{2}\right)$

Figure 3. ARC-C defines significant interactions between regulatory elements, candidate regulators, and a role for BLMP-1 in mediating interactions. ( $A$ ) Types of elements and types of interactions involved in the 14,901 significant interactions. (B) Interaction distances of the significant interactions, separated by element type. (C) Expression correlation between pairs of genes with linked promoters compared to randomly paired genes in the set. (Left) All pairs of linked genes $(n=5142)$; (middle) pairs for which both genes are in the bottom $30 \%$ of CV values (wide expression; $n=2224$ ); (right) pairs for which both genes are in the top $70 \%$ of $C V$ values (regulated expression; $n=879$ ). ( $(D$, left) Contact frequency between binding sites of indicated proteins; (right) change in contact frequency in blmp-1 mutants compared to wild type $\left(\log _{2}\right)$. Interactions among blmp-1 down targets are significantly reduced. See Methods for details and Supplemental Table S5 for data. 
formation (Rao et al. 2014, 2017); C. elegans does not contain an ortholog of boundary factor CTCF, which functions with cohesin in mammals (Heger et al. 2009). We also observed significant enrichment for interactions between binding sites of subunits of the condensin II and Retinoblastoma/DREAM complexes, 33 transcription factors, and other chromatin regulators (Fig. 3D). These proteins are strong candidates for involvement in mediating chromatin interactions.

To evaluate the ability of ARC-C to detect changes in regulatory interactions, we chose to analyze BLMP-1, a TF for which binding sites significantly interact (Fig. 3D). blmp-1 encodes a transcription factor important for hypodermal, vulval, and gonadal development (Horn et al. 2014; Huang et al. 2014; Yang et al. 2015). We performed ARC-C in L3 stage blmp-1 mutants and asked whether chromatin interactions at pairs of TF and chromatin regulator binding sites were altered compared to wild-type L3 larvae. No significant changes in interaction frequency were detected for the analyzed factors, including the full set of BLMP-1 binding sites (Fig. 3D; Supplemental Table S5). We next analyzed direct BLMP1 targets, defined as the subsets of BLMP-1 binding regions associated with a gene that was up-regulated or down-regulated in blmp-1 mutants (Supplemental Table S6). Chromatin interactions between targets down-regulated in $b l m p-1$ mutants were significantly reduced (Fig. 3D; down targets in Supplemental Table S5), showing that ARC-C can detect specific changes in mutants. The results suggest that BLMP-1 may promote spatial proximity between targets that require it for expression, for example, by directly mediating interactions. Valid coverage at the down-regulated targets is also reduced 1.2-fold in blmp-1 mutants, suggesting that BLMP-1 contributes to facilitating open chromatin at these sites.

\section{Discussion}

In its present form, ARC-C works well for profiling chromatin interactions in relatively small genomes, because sequencing 200 million fragments per duplicate library produces enough cis-informative read pairs for profiling architecture and regulatory element interactions. For application to larger mammalian genomes, an enrichment step for ligation events (e.g., through biotin tagging) would be beneficial.

Here, we used ARC-C in whole animals, so the cell types from which the detected interactions came are unknown. In addition, interactions that occur in a small number of cells are likely to have been missed. The future application of ARC-C to specific purified cells would address these issues, allowing in vivo investigation of cell type-specific architecture.

In conclusion, ARC-C provides a new ability to study genome topology and regulatory interactions at high resolution in a single genome-wide assay. Our application of ARC-C in C. elegans revealed unappreciated domain and compartment structure and proteins that are candidates for mediating organization. The use of ARC-C and study of these candidates should accelerate studies of transcriptional regulation and the relationship with genome architecture.

\section{Methods}

\section{Worm strains and culture}

C. elegans strains were maintained at $20^{\circ} \mathrm{C}$ as previously described (Brenner 1974). The following strains were used: Bristol N2 (wild type), YJ55 blmp-1(tm548) (Huang et al. 2014), and GW638 met2(n4256) set-25(n5021) (Towbin et al. 2012).

\section{Worm growth}

Strains for ARC-C and ChIP-seq were grown in liquid culture at $20^{\circ} \mathrm{C}$ using standard S-basal medium with HB101 bacteria. Animals were first grown to the adult stage, bleached to obtain embryos, and the embryos hatched without food in M9 buffer for $24 \mathrm{~h}$ at $20^{\circ} \mathrm{C}$ to obtain synchronized starved L1 larvae. L1 larvae were grown in a further liquid culture at $20^{\circ} \mathrm{C}$ then harvested at the L3 stage. Worms were collected, washed in M9 buffer, floated on sucrose, washed again in M9, then frozen into small pellets by dripping worm slurry into liquid nitrogen, which was stored at $-80^{\circ} \mathrm{C}$ until use.

\section{ChIP-seq}

JMJD-2, SCC-1, ZFP-1, BLMP-1, TOP-2, and SIN-3 chromatin immunoprecipitations in L3 larvae and library preparations were conducted as in McMurchy et al. (2017). Antibodies used were JMJD-2 (antibody Q3951; this study, raised against amino acids 50-149), SCC-1 (Novus antibody 29510002, lot Q0835; raised against amino acids 421-520); ZFP-1 (antibody Q2059; this study, raised against amino acids 1-100), BLMP-1 (antibody Q2919; this study, raised against amino acids 43-142), TOP-2 (antibody Q5515; this study, raised against aa317-437), and SIN-3 (antibody Q6013; Beurton et al. 2019).

\section{Differential expression analysis of met-2 set-25 mutant}

Wild-type and met-2 set- 25 mutant worms were grown at $20^{\circ} \mathrm{C}$ on NGM plates, harvested at the L3 stage, and flash frozen in liquid nitrogen. RNA was extracted from frozen worms using TriPure (Roche) and purified with the Zymo Research RNA Clean and Concentrator kit (R1013) after DNase I digestion. Libraries were prepared with the TruSeq RNA Library Prep Kit (Illumina). Reads were aligned to genome assembly ce10 with gene annotation WS235 using STAR (Dobin et al. 2013). Read counts per gene were generated by HTSeq and genes differentially expressed in met-2 set- 25 relative to wild type identified using DESeq2 (Love et al. 2014), requiring adjusted $P<0.05$ (Supplemental Table S1). Genes were annotated as being marked by $\mathrm{H} 3 \mathrm{~K} 9 \mathrm{me} 2$ or $\mathrm{H} 3 \mathrm{~K} 9 \mathrm{me} 3$ if their average signal was $1.5 \times$ above the median, using L3 ChIP-seq data sets available under the NCBI Gene Expression Omnibus (GEO; https://www .ncbi.nlm.nih.gov/geo/) accession numbers GSE49728 for H3K9me2 and GSE49720 for H3K9me3 (Ho et al. 2014).

\section{Differential expression analysis of blmp-1 mutant}

Raw RNA-seq data of wild-type and blmp-1(tm548) mutants at L3 stage were obtained from GEO (GSE55225) (Horn et al. 2014). Reads were aligned to genome assembly ce10 with gene annotation WS235 using STAR (Dobin et al. 2013). Read counts per gene were generated by featureCounts, and genes differentially expressed in blmp-1 relative to wild type were identified using DESeq2 (Love et al. 2014), requiring adjusted $P<0.05$ and absolute fold change $>1.5$. Genes significantly up-regulated or down-regulated in blmp-1 mutants compared to wild type and for which a BLMP-1 ChIP-seq peak overlapped one of its assigned regulatory elements were defined as up-regulated "up" or down-regulated "down" BLMP-1 targets (Supplemental Table S6).

\section{ARC-C library preparation}

A key element of ARC-C is to digest nuclei with DNase I in situ under conditions in which cutting results in maximal enrichment of informative read pairs at accessible chromatin. This concentration

\section{Genome Research}

www.genome.org 
should be empirically determined for the sample type of interest. For C. elegans nuclei, we found that 50-200 units/mL DNase I gives optimal recovery of informative read pairs using the digestion conditions indicated below (Supplemental Fig. S1).

Frozen worm pellets were ground into a fine powder in which worms were broken into approximately 10 fragments. Then $1 \mathrm{~mL}$ of worm powder was fixed in $10 \mathrm{~mL}$ of $1 \%$ formaldehyde in PBS for 10 min at room temperature (RT) with gentle shaking then quenched for $5 \mathrm{~min}$ with a final concentration of $125 \mathrm{mM}$ glycine. Fixed worm fragments were then washed with Buffer A $(340 \mathrm{mM}$ sucrose, $15 \mathrm{mM}$ Tris- $\mathrm{HCl}$ at $\mathrm{pH} 7.5,2 \mathrm{mM} \mathrm{MgCl}_{2}, 0.5 \mathrm{mM}$ spermidine, $0.15 \mathrm{mM}$ spermine, $1 \mathrm{mM}$ DTT, protease inhibitors), resuspended in $7 \mathrm{~mL}$ Buffer A, then the material dounced 20 strokes in a $7 \mathrm{~mL}$ stainless steel tissue grinder (VWR 432-5005). The dounced material was spun at $100 \mathrm{~g}$ for $5 \mathrm{~min}$; the supernatant, which contains nuclei, was transferred to a new tube. The pellet was resuspended in Buffer A and again dounced with 20 strokes. After spinning, the two supernatants containing nuclei were pooled.

Aliquots of 10 million fixed nuclei were spun down at $1000 \mathrm{~g}$ and resuspended in $200 \mu \mathrm{L}$ of $1 \times$ DNase buffer (Roche), and chromatin was digested with 50 and/or 100 units/mL DNase I for 10 $\min$ at $25^{\circ} \mathrm{C}$. The reactions were then quenched with a final concentration of $25 \mathrm{mM}$ EDTA and $5 \mathrm{mM}$ Tris at $\mathrm{pH}$ 7.5. Nuclei were washed twice with $1 \mathrm{~mL}$ of ice-cold Nuclear Washing Buffer (340 $\mathrm{mM}$ sucrose, $15 \mathrm{mM}$ Tris- $\mathrm{HCl}$ at $\mathrm{pH} 7.5,25 \mathrm{mM}$ EDTA, $0.5 \mathrm{mM}$ spermidine, $0.15 \mathrm{mM}$ spermine, $1 \mathrm{mM}$ DTT, protease inhibitors). Nuclei were then resuspended in $100 \mu \mathrm{L}$ of end repair mastermix $(10 \mu \mathrm{L} 10 \times$ NEB End Repair Buffer, $5 \mu \mathrm{L}$ NEB End Repair Enzyme Mix, $85 \mu \mathrm{L} \mathrm{H}_{2} \mathrm{O}$ ) and incubated for $30 \mathrm{~min}$ at $20^{\circ} \mathrm{C}$ with rotation. Thereafter, $400 \mu \mathrm{L}$ of ligation master-mix was added (40 $\mu \mathrm{L} 10 \times$ ligation buffer, $5 \mu \mathrm{L}$ T4 DNA ligase [400,000 units/ $\mathrm{mL}$ ], $355 \mu \mathrm{L} \mathrm{H}_{2} \mathrm{O}$ ) and the mixture was incubated overnight at $4^{\circ} \mathrm{C}$ with rotation. Nuclei were pelleted, resuspended in $50 \mu \mathrm{L}$ of tagmentation master-mix $\left(22.5 \mu \mathrm{L} \mathrm{H}_{2} \mathrm{O}, 25 \mu \mathrm{L} 2 \times\right.$ Nextera TD buffer, $2.5 \mu \mathrm{L}$ Nextera Tn5 transposase), and incubated for $30 \mathrm{~min}$ at $37^{\circ} \mathrm{C}$. Next, $5 \mu \mathrm{L}$ of $1 \%$ SDS and $2 \mu \mathrm{L}$ of NEB Proteinase $\mathrm{K}$ [800 units $/ \mathrm{mL}$ ] was added and left for $15 \mathrm{~min}$ at $65^{\circ} \mathrm{C}$ before DNA from the mixture was purified using Qiagen MinElute columns. Large fragments ( $>500 \mathrm{bp}$ ) were removed from the purified DNA using two rounds of $0.6 \mathrm{vol}$ AMPure XP beads. Before PCR amplification, a test qPCR amplification using 1/20th of the input DNA was performed, and the cycle number for PCR was determined by the midpoint of the exponential phase of the amplification curve. The resultant DNA was amplified with NEBNext Ultra II Q5 Master-mix under the following PCR conditions: for $5 \mathrm{~min}$ at $72^{\circ} \mathrm{C}$, for $30 \mathrm{sec}$ at $98^{\circ} \mathrm{C}$, and cycling for $10 \mathrm{sec}$ at $98^{\circ} \mathrm{C}$, for $30 \mathrm{sec}$ at $63^{\circ} \mathrm{C}$, and for $1 \mathrm{~min}$ at $72^{\circ} \mathrm{C}$ using the determined cycle number. The library was size-selected with AMPure XP beads to a final range of 200-700 bp (insert size: $\sim 70-570 \mathrm{bp}$ ). Before sequencing, libraries were quality tested by qPCR at the gap-3 promoter. DNase I overdigestion or underdigestion led to poor enrichment of regulatory elements. Good libraries generally had gap-3 promoter enrichment values of greater than fourfold. Libraries were sequenced on the Illumina platform paired-end (100 bp). Four libraries were sequenced from wild-type L3 larvae (three biological replicates and two technical replicates; separate ARC-C libraries made from the same biological material: N2rep1, N2-rep2a, N2-rep2b, and N2-rep3 in Supplemental Fig. $\mathrm{S} 2 \mathrm{~A}$ ), and two biological replicate libraries were sequenced for both blmp-1 and met-2 set-25 L3 larvae.

\section{Processing ARC-C data}

ARC-C libraries were sequenced 62-150 bp (100 bp for most samples) from both ends. Supplemental Figure S2 lists libraries ana- lyzed in the paper and sequencing statistics. Adapter sequence was trimmed by cutadapt (Martin 2011), and sequences with $>20$ bp remaining were removed. Each sequenced end was aligned independently to the ce10 reference genome using BWA-MEM (Li 2013), which allows split-read alignment using the default parameters. The two aligned ends (or the $5^{\prime}$ segment for split alignments) were then paired. We required both ends of a pair to align uniquely and with high confidence (mapping quality $\geq 30$ and number of mismatches $\leq 2$ ) to the nuclear genome and outside modENCODE backlisted-regions. PCR duplicates were next removed by sambamba markdup. The remaining read pairs were regarded as valid read pairs. Valid read pairs mapping to different chromosomes or $>600 \mathrm{bp}$ apart on the same chromosome were regarded as trans- or cis-informative read pairs, respectively. The 600-bp threshold was established by comparing the proportions of the four possible end alignment orientation configurations (forward-forward, forward-reverse, reverse-forward, and reverse-reverse) as a function of mapping distance. The vast majority of pairs mapping $<500 \mathrm{bp}$ apart were in the forward-reverse configuration (nonligated fragments), whereas $>600$ bp the proportions were stably at $25 \%$ each (Supplemental Fig. S12).

Contact maps were made from informative read pairs by binning the genome into fixed-width $(1,5,10$, or $50 \mathrm{~kb})$ nonoverlapping bins and counting the number of read pairs between each pair of bins. The maps were then normalized by matrix balancing using the Knight-Ruiz algorithm (Knight and Ruiz 2013). Concordance between replicate maps was assessed by GenomeDISCO (Ursu et al. 2018). The lower resolution 50-kb maps were used for whole-chromosome visualization. The 1-, 5-, and 10-kb maps were further corrected for distance-dependent background contact frequency by dividing the spline-smoothed average contact frequency given the distance to the diagonal. These higher resolution maps were used for aggregated contact analysis (see below) of nuclear factor binding sites and chromatin domain/compartment, respectively.

\section{Processing $\mathrm{Hi}-\mathrm{C}$ data}

Raw FASTQ files of wild-type mixed embryo Hi-C data (Crane et al. 2015) were downloaded from the NCBI Sequence Read Archive (SRA; https://www.ncbi.nlm.nih.gov/sra) accession number SRX77040 and processed using HiCUP v0.5.9 (Wingett et al. 2015), which filters for same fragment (circularized, dangling ends, internal), religation, wrong size, contiguous sequence, and removes duplicate read pairs. We additionally required mapQ $\geq$ 30 and number of mismatches $\leq 2$ from both reads, that none of the reads overlapped modENCODE blacklisted regions, and a minimum distance of $600 \mathrm{bp}$ between the two read pairs to be consistent with the processing of ARC-C data. In the end, 25,460,294 read pairs passed all filters, of which $17,100,808$ have both reads mapping to the same chromosome.

\section{Calling significant interactions}

We first segmented the genome into bins of $\sim 500$ bp using the following procedure. We first took annotated regulatory elements (Jänes et al. 2018) ( $n=42,245)$ and expanded them to 500 bp or until neighboring intervals began to touch; a small number of elements that were within $100 \mathrm{bp}$ were merged first. The rest of the genome was covered with evenly placed 500-bp nonoverlapping fixed-width intervals; hence, the entire genome was covered by a combined set of 192,257 intervals of average size (494 bp). We used this procedure instead of generating fixed nonoverlapping bins to avoid individual Res being split into two bins.

Before assessing the significance of chromatin interactions in Hi-C or other chromatin interaction data sets, inherent bias 
resulting from uneven coverage and physical proximity need to be accounted for. A difference in ARC-C data compared to that of Hi-C is the specific overrepresentation of open chromatin. The observed enrichments at these regions are a compound effect of enriching the regions themselves and of their being enriched by virtue of linkage to another region of open chromatin (true signals). To address this and avoid normalizing out interaction signals, we developed an approach similar to that used for Capture Hi-C, which has similar coverage biases and used "off-peak" interaction frequency to account for coverage (Cairns et al. 2016). We modeled the number of read pairs linking two intervals as a random variable following a binomial distribution parameterized by an expected contact frequency determined by unevenness of coverage and distance between the interval (Ay et al. 2014), as described next.

For every interval $i \in I$, the number of $c i s$-informative read pairs $c_{c i s, i}$ were counted. Intervals in the top $10 \%$ of the coverage distribution were regarded as peaks, and intervals in the bottom $10 \%$ were removed. An off-peak cis-informative coverage $c_{\text {offpeak }, i}$ was calculated for every kept interval, counting the number of contacts not involving peak intervals. We calculated a scaling factor for the interval's representation/visibility as $v_{i}=\left(c_{\text {offpeak }, i} /\right.$ median $\left.\left(c_{\text {offpeak, }}\right)\right)^{0.87}$ (see below for derivation of the exponent). Chromosome-wide average distance-dependent contact frequency $F(d)$ in the distance range of $1 \mathrm{~kb}$ to $1 \mathrm{Mb}$ was modeled by fitting a spline function in a two-pass process (following Fit-Hi-C) (Ay et al. 2014). For every pair of intervals with a distance between $1 \mathrm{~kb}$ and $1 \mathrm{Mb}$, an expected contact frequency was calculated given the distance and the visibility of each interval as $f_{i, j}=v_{i} v_{j} F\left(d_{i, j}\right)$. Given the total number of cis-informative contacts $(N)$ of the chromosome, we considered a null distribution in the form of a binomial, where the observed number of contacts, $n_{i, j} \sim \operatorname{binomial}\left(N, f_{i, j}\right)$. Significant interactions were called at an FDR level of 0.05 and were post-filtered requiring support by more than five read pairs.

The appropriate correction factors for adjusting the representation bias, $X$, should be able to transform the unnormalized contact matrix $A$ into a normalized contact matrix $B$ by $B=\operatorname{diag}(X)^{*}$ $A^{*} \operatorname{diag}(X)$, such that each row or column of $B$ sums up to the same value, thus eliminating the unevenness of representation across different bins. It has been well-established that a correct set of factors can be found using the method "matrix balancing" (MB) (Imakaev et al. 2012; Rao et al. 2014), and an efficient algorithm has been developed (Knight and Ruiz 2013; Rao et al. 2014). Our goal is to find correction factors that enable transformation into a normalized contact matrix in which each bin has the same off-peak coverage. Because an efficient algorithm for solving this problem at high resolution is not yet available, we aimed to find an approximate solution. Two measures, namely, the reciprocal of coverage $(c)$ and square root coverage $\left(c^{0.5}\right)$, have been proposed for use in the place of MB-derived correction factors (Rao et al. 2014). It was reported that the former can overcorrect, whereas the latter gives good approximation to MB-derived correction factors (Rao et al. 2014). We examined correlation between MB-derived correction factors and the reciprocal of different exponents of coverage (Supplemental Fig. S13) and found that the reciprocal of $c$ indeed overcorrects, but the reciprocal of square root coverage undercorrects. The most accurate approximation is achieved at around an exponent of 0.87 . Therefore, we used the reciprocal of $\left(c_{\text {offpeak }}\right)^{0.87}$ as the correction factor.

\section{Aggregated contact analyses}

A contact is defined as the region in the contact map that connects a pair of genomic locations. Aggregated contact analysis is a method of visualizing the average contact frequency of a group of many contacts together with local contact frequency (Rao et al. 2014).
We applied this method to both small genomic regions, such as nuclear factor ChIP-seq binding sites (NFBS), and to larger intervals, such as chromatin domains. We normalized contact maps using matrix balancing (Imakaev et al. 2012) to account for coverage bias and removed distance-dependent background. In NFBS analysis, we used normalized and background-frequency-corrected contact maps of $1-\mathrm{kb}$ resolution. For each NF, up to 50,000 contacts were randomly sampled from all possible cis-contacts among its binding sites within a distance range from $20 \mathrm{~kb}$ to $1 \mathrm{Mb}$, and local maps of $21 \times 21$ bins centered at the contacts were extracted and aggregated. For the case of BLMP-1 regulated targets, all possible cis-contacts between BLMP-1 binding sites that involves a BLMP-1 regulated target were aggregated (i.e., at least one end of the contact is at a BLMP- 1 regulated target). The $\log _{2}$ fold change of the central point over the mean of the rest of the points in the aggregated map was calculated to measure the relative increase in contact frequency over local background. To assess statistical significance while controlling for accessibility and the distance between the pair of NFBSs, 1000 sets of random contacts were generated, each containing the same number of contacts with matching accessibility and distance as the NFBS contacts. Each of the 1000 random sets was aggregated and a relative increase in contact frequency was calculated in the same way as the NFBS set, forming a distribution of values against which the NFBS value was compared and a $P$-value generated, which was corrected for multiple testing using the FDR method. The rex-rex APA analyses used normalized and background-frequency-corrected contact maps of 10$\mathrm{kb}$ resolution and a distance range of $100 \mathrm{~kb}$ to $4 \mathrm{Mb}$. Data sets (Kranz et al. 2013; Araya et al. 2014; Ho et al. 2014; Latorre et al. 2015; Wiesenfahrt et al. 2016; McMurchy et al. 2017; Kudron et al. 2018) are listed in Supplemental Table S7. Processed and curated modENCODE ChIP-seq peaks (Araya et al. 2014; Kudron et al. 2018) were obtained from Jänes et al. (2018). For ChIP-seq performed in this paper (JMJD-2, SCC-1, ZFP-1, BLMP-1, TOP-2, and SIN-3), peaks were called for each replicate separately as for accessible sites in Jänes et al. (2018) using YAPC (https://github.com/ jurgjn/yapc), except for BLMP-1, which used MACS2 (Feng et al. 2012). ChIP-seq peaks were combined by IDR (Li et al. 2011) with a $P$-value threshold of 0.01 . We removed intervals that are highly occupied target ("HOT") because such binding events are thought to represent non-sequence-specific TF binding or ChIP artifacts (Gerstein et al. 2010; Kudron et al. 2018). This was defined as the top $20 \%$ of peak intervals ranked by the number factors in which the interval is called (effectively removing intervals called in 12 or more factors). We only considered data sets having at least 300 peaks following filtering.

For domain analyses, we used normalized and backgroundfrequency-corrected contact maps of 5-kb resolution. L3 stage chromatin domains are from Evans et al. (2016). Regions annotated as active and border were merged to generate the active domains used here; the H3K27me3 domains are those termed "regulated." Informative reads mapped at similar levels to active and H3K27me3 domains: 10.6 million (44.4\%) of all informative reads mapped to active domains (39.0 $\mathrm{Mb}, 38.9 \%$ of the genome), whereas 8.1 million (33.8\%) mapped to H3K27me3 domains ( $42.3 \mathrm{Mb}, 42.1 \%$ of the genome). The rest of the reads mapped to Chromosome X, for which active and H3K27me3 domains were not mapped. The small (1.42-fold) bias in coverage was normalized by the matrix balancing that was performed on the contact frequency matrix to normalize the differences in sequencing coverage across all genomic bins.

We tested for TADs by assessing intra-domain contacts. For each type of domain (active or H3K27me3), maps of each contact region containing a domain of at least $5 \mathrm{~kb}$ together with up to 25 $\mathrm{kb}$ of the flanking domains of opposite type were extracted and

\section{Genome Research}

www.genome.org 
aggregated. Where neighboring domains were $<25 \mathrm{~kb}$, only the domain was extracted. The aggregated contact was scaled to a square of $9 \times 9$ bins, and the flanking intervals were scaled to five bins wide. The $\log _{2}$ fold change of the mean of the central $9 \times 9$ square over the mean of the four neighboring $5 \times 10$ rectangles on top, bottom, left, and right was calculated to measure the relative strength in contact frequency with $P$-values generated by $t$ test. We tested for compartments using the same approach, by assessing all possible pairs of inter-domain contacts in the range from $50 \mathrm{~kb}$ to $2 \mathrm{Mb}$. To compare domain and compartment strength in met-2 set-25 mutants relative to wild type, the fold change in percentage difference between central block and side blocks across strains were calculated.

To test the effect of local H3K9 methylation on TAD and compartment strength, we separated active and H3K27me3 domains into highly and lowly marked sets. For each active and H3K27me3 domain, the average coverage of H3K9me1, H3K9me2, and H3K9me3 was calculated using wild-type L3 ChIP-seq data (Ho et al. 2014). For each modification, active and H3K27me3 domains in the top $25 \%$ of signal were defined as H3K9me1, H3K9me2, or H3K9me3 high domains, and the remainder as low domains. Aggregated contact analysis of TADs and compartments was performed using wild-type and met- 2 set-25 ARC-C data for each of the domain sets as described above.

\section{Aggregated contact analysis of blmp-1 mutant ARC-C data}

We used the procedure described above for wild type to measure the relative contact frequency between NFBSs in blmp-1 ARC-C data. To normalize overall open chromatin measurements between blmp-1 and wild-type maps, we first fitted blmp-1 ACA measures as a linear function of the respective measures in wild type, that is, $\log _{2}\left(\mathrm{blmp} 1 \_\mathrm{FC}\right)=\mathrm{a}+\mathrm{b}{ }^{*} \log _{2}$ (N2_FC). The residuals were then used to measure the difference in contact frequency in blmp-1 relative to wild type. Statistical significance was assessed by 10,000-time bootstrapping the distribution of residuals, and $P$-values were adjusted by FDR (Supplemental Table S5).

\section{Expression correlation between interacting promoters}

For genes linked by promoter-promoter interactions, we calculated Pearson correlation coefficients of gene expression across cell types using data from Cao et al. (2017). For pairs involving bidirectional promoters, the gene pair with highest correlation was chosen. For each gene, we also calculated a coefficient of variation of gene expression (CV) across the cell types (Cao et al. 2017) as a measure of tissue-biased expression. Genes with similar expression across cell types have low gene expression $\mathrm{CV}$ values and those with tissue-biased expression have high CV values. In Figure 3C, we assessed expression correlation between all linked pairs of genes $(n=5124)$, linked genes for which both were in the bottom $30 \%$ of all CV values $(n=2224)$, and linked genes for which both were in the top $70 \%$ of $C V$ values $(n=879)$. To assess the statistical significance of the correlations, we generated control sets by sampling the same number of random pairs using genes from the respective linked gene sets while requiring the distribution of distance between the random pairs to match that of the observed set. Difference in correlation between the observed and the random control set was tested using a $t$-test: all $\left(P=7.77 \times 10^{-27}\right)$, bottom $30 \%\left(P=4.38 \times 10^{-06}\right)$, and top $70 \%\left(P=5.69 \times 10^{-20}\right)$.

\section{Data access}

The ARC-C, ChIP-seq, and RNA-seq data generated in this study have been submitted to the NCBI Gene Expression Omnibus
(GEO; https://www.ncbi.nlm.nih.gov/geo/) under accession number GSE144673. The code used in this paper is available at GitHub (https://github.com/nh3/ctk) and as Supplemental Code.

\section{Competing interest statement}

The authors declare no competing interests.

\section{Acknowledgments}

We thank K. Harnish for sequencing, P. Kolasinska-Zwierz and M. Herrmann for help with ChIP experiments, and F. Carelli and R. Durbin for comments on the manuscript. The work was supported by a Wellcome Trust Senior Research Fellowship to J.A. (101863) and an A-star fellowship to W.Q.S. We also acknowledge core support from the Wellcome Trust (092096) and Cancer Research UK (C6946/A14492).

Author contributions: W.Q.S. and J.A. designed the study; W.Q.S. performed the research, with contributions from A.A. and Y.D.; N.H. analyzed the data with contributions from P.S.; N.H., W.Q.S., and J.A. wrote the manuscript; all authors reviewed and edited the manuscript.

\section{References}

Ahringer J, Gasser SM. 2018. Repressive chromatin in Caenorhabditis elegans: establishment, composition, and function. Genetics 208: 491-511. doi:10.1534/genetics.117.300386

Araya CL, Kawli T, Kundaje A, Jiang L, Wu B, Vafeados D, Terrell R, Weissdepp P, Gevirtzman L, Mace D, et al. 2014. Regulatory analysis of the C. elegans genome with spatiotemporal resolution. Nature 512: 400-405. doi:10.1038/nature13497

Ay F, Bailey TL, Noble WS. 2014. Statistical confidence estimation for Hi-C data reveals regulatory chromatin contacts. Genome Res 24: 999-1011. doi:10.1101/gr.160374.113

Beurton F, Stempor P, Caron M, Appert A, Dong Y, Chen R, Cluet D, Couté Y, Herbette M, Huang N, et al. 2019. Physical and functional interaction between SET1/COMPASS complex component CFP-1 and a Sin3S HDAC complex in C. elegans. Nucleic Acids Res 47: 11164-11180. doi:10.1093/nar/gkz880

Bian Q, Anderson EC, Yang Q, Meyer BJ. 2020. Histone H3K9 methylation promotes formation of genome compartments in Caenorhabditis elegans via chromosome compaction and perinuclear anchoring. Proc Natl Acad Sci 117: 11459-11470. doi:10.1073/pnas.2002068117

Brejc K, Bian Q, Uzawa S, Wheeler BS, Anderson EC, King DS, Kranzusch PJ, Preston CG, Meyer BJ. 2017. Dynamic control of X chromosome conformation and repression by a histone H4K20 demethylase. Cell 171: 85102.e23. doi:10.1016/j.cell.2017.07.041

Brenner S. 1974. The genetics of Caenorhabditis elegans. Genetics 77: 71-94. doi:10.1093/genetics/77.1.71

Buenrostro JD, Giresi PG, Zaba LC, Chang HY, Greenleaf WJ. 2013. Transposition of native chromatin for fast and sensitive epigenomic profiling of open chromatin, DNA-binding proteins and nucleosome position. Nat Methods 10: 1213-1218. doi:10.1038/nmeth.2688

Cairns J, Freire-Pritchett P, Wingett SW, Várnai C, Dimond A, Plagnol V, Zerbino D, Schoenfelder S, Javierre BM, Osborne C, et al. 2016. CHiCAGO: robust detection of DNA looping interactions in Capture Hi-C data. Genome Biol 17: 127. doi:10.1186/s13059-016-0992-2

Cao J, Packer JS, Ramani V, Cusanovich DA, Huynh C, Daza R, Qiu X, Lee C, Furlan SN, Steemers FJ, et al. 2017. Comprehensive single-cell transcriptional profiling of a multicellular organism. Science 357: 661-667. doi:10.1126/science.aam8940

Crane E, Bian Q, McCord RP, Lajoie BR, Wheeler BS, Ralston EJ, Uzawa S, Dekker J, Meyer BJ. 2015. Condensin-driven remodelling of X chromosome topology during dosage compensation. Nature 523: 240-244. doi:10.1038/nature 14450

Davies JO, Oudelaar AM, Higgs DR, Hughes JR. 2017. How best to identify chromosomal interactions: a comparison of approaches. Nat Methods 14: $125-134$. doi:10.1038/nmeth.4146

Dekker J, Heard E. 2015. Structural and functional diversity of topologically associating domains. FEBS Lett 589: 2877-2884. doi:10.1016/j.febslet .2015 .08 .044

Dekker J, Rippe K, Dekker M, Kleckner N. 2002. Capturing chromosome conformation. Science 295: 1306-1311. doi:10.1126/science.1067799 
Dekker J, Marti-Renom MA, Mirny LA. 2013. Exploring the three-dimensional organization of genomes: interpreting chromatin interaction data. Nat Rev Genet 14: 390-403. doi:10.1038/nrg3454

Dixon JR, Selvaraj S, Yue F, Kim A, Li Y, Shen Y, Hu M, Liu JS, Ren B. 2012 Topological domains in mammalian genomes identified by analysis of chromatin interactions. Nature 485: 376-380. doi:10.1038/ nature 11082

Dobin A, Davis CA, Schlesinger F, Drenkow J, Zaleski C, Jha S, Batut P, Chaisson M, Gingeras TR. 2013. STAR: ultrafast universal RNA-seq aligner. Bioinformatics 29: 15-21. doi:10.1093/bioinformatics/bts635

Evans KJ, Huang N, Stempor P, Chesney MA, Down TA, Ahringer J. 2016. Stable Caenorhabditis elegans chromatin domains separate broadly expressed and developmentally regulated genes. Proc Natl Acad Sci 113: E7020-E7029. doi:10.1073/pnas.1608162113

Falk M, Feodorova Y, Naumova N, Imakaev M, Lajoie BR, Leonhardt H, Joffe B, Dekker J, Fudenberg G, Solovei I, et al. 2019. Heterochromatin drives compartmentalization of inverted and conventional nuclei. Nature 570: 395-399. doi:10.1038/s41586-019-1275-3

Feng J, Liu T, Qin B, Zhang Y, Liu XS. 2012. Identifying ChIP-seq enrichment using MACS. Nat Protoc 7: 1728-1740. doi:10.1038/nprot.2012 .101

Gaydos LJ, Rechtsteiner A, Egelhofer TA, Carroll CR, Strome S. 2012. Antagonism between MES-4 and Polycomb repressive complex 2 promotes appropriate gene expression in C. elegans germ cells. Cell Rep 2: 1169-1177. doi:10.1016/j.celrep.2012.09.019

Gerstein MB, Lu ZJ, Van Nostrand EL, Cheng C, Arshinoff BI, Liu T, Yip KY, Robilotto R, Rechtsteiner A, Ikegami K, et al. 2010. Integrative analysis of the Caenorhabditis elegans genome by the modENCODE project. Science 330: $1775-1787$. doi:10.1126/science.1196914

Heger P, Marin B, Schierenberg E. 2009. Loss of the insulator protein CTCF during nematode evolution. BMC Mol Biol 10: 84. doi:10.1186/1471 2199-10-84

Ho JW, Jung YL, Liu T, Alver BH, Lee S, Ikegami K, Sohn KA, Minoda A, Tolstorukov MY, Appert A, et al. 2014. Comparative analysis of metazoan chromatin organization. Nature 512: 449-452. doi:10.1038/ nature 13415

Horn M, Geisen C, Cermak L, Becker B, Nakamura S, Klein C, Pagano M, Antebi A. 2014. DRE-1/FBXO11-dependent degradation of BLMP-1/ BLIMP-1 governs $C$. elegans developmental timing and maturation. Dev Cell 28: 697-710. doi:10.1016/j.devcel.2014.01.028

Huang TF, Cho CY, Cheng YT, Huang JW, Wu YZ, Yeh AY, Nishiwaki K, Chang SC, Wu YC. 2014. BLMP-1/Blimp-1 regulates the spatiotemporal cell migration pattern in C. elegans. PLoS Genet 10: e1004428. doi:10 .1371/journal.pgen.1004428

Hughes JR, Roberts N, McGowan S, Hay D, Giannoulatou E, Lynch M, De Gobbi M, Taylor S, Gibbons R, Higgs DR. 2014. Analysis of hundreds of cis-regulatory landscapes at high resolution in a single, high-throughput experiment. Nat Genet 46: 205-212. doi:10.1038/ng.2871

Ikegami K, Egelhofer TA, Strome S, Lieb JD. 2010. Caenorhabditis elegans chromosome arms are anchored to the nuclear membrane via discontinuous association with LEM-2. Genome Biol 11: R120. doi:10.1186/ gb-2010-11-12-r120

Imakaev M, Fudenberg G, McCord RP, Naumova N, Goloborodko A, Lajoie BR, Dekker J, Mirny LA. 2012. Iterative correction of Hi-C data reveals hallmarks of chromosome organization. Nat Methods 9: 999-1003. doi:10.1038/nmeth. 2148

Jänes J, Dong Y, Schoof M, Serizay J, Appert A, Cerrato C, Woodbury C, Chen R, Gemma C, Huang N, et al. 2018. Chromatin accessibility dynamics across C. elegans development and ageing. eLife 7: e37344. doi:10.7554/eLife.37344

Knight PA, Ruiz D. 2013. A fast algorithm for matrix balancing. IMA J Numer Anal 33: 1029-1047. doi:10.1093/imanum/drs019

Kranz AL, Jiao CY, Winterkorn LH, Albritton SE, Kramer M, Ercan S. 2013. Genome-wide analysis of condensin binding in Caenorhabditis elegans. Genome Biol 14: R112. doi:10.1186/gb-2013-14-10-r112

Kudron MM, Victorsen A, Gevirtzman L, Hillier LW, Fisher WW, Vafeados D, Kirkey M, Hammonds AS, Gersch J, Ammouri H, et al. 2018. The ModERN resource: genome-wide binding profiles for hundreds of Drosophila and Caenorhabditis elegans transcription factors. Genetics 208: 937-949. doi:10.1534/genetics.117.300657

Latorre I, Chesney MA, Garrigues JM, Stempor P, Appert A, Francesconi M, Strome S, Ahringer J. 2015. The DREAM complex promotes gene body H2A.Z for target repression. Genes Dev 29: 495-500. doi:10.1101/gad .255810 .114

Li H. 2013. Aligning sequence reads, clone sequences and assembly contigs with BWA-MEM. arXiv:1303.3997v2.

Li Q, Brown JB, Huang H, Bickel PJ. 2011. Measuring reproducibility of highthroughput experiments. Ann Appl Stat 5: 1752-1779. doi:10.1214/11AOAS466
Lieberman-Aiden E, van Berkum NL, Williams L, Imakaev M, Ragoczy T, Telling A, Amit I, Lajoie BR, Sabo PJ, Dorschner MO, et al. 2009. Comprehensive mapping of long-range interactions reveals folding principles of the human genome. Science 326: 289-293. doi:10.1126/sci ence. 1181369

Liu T, Rechtsteiner A, Egelhofer TA, Vielle A, Latorre I, Cheung MS, Ercan S, Ikegami K, Jensen M, Kolasinska-Zwierz P, et al. 2011. Broad chromosomal domains of histone modification patterns in C. elegans. Genome Res 21: 227-236. doi:10.1101/gr.115519.110

Love MI, Huber W, Anders S. 2014. Moderated estimation of fold change and dispersion for RNA-seq data with DESeq2. Genome Biol 15: 550. doi:10.1186/s13059-014-0550-8

Ma W, Ay F, Lee C, Gulsoy G, Deng X, Cook S, Hesson J, Cavanaugh C, Ware CB, Krumm A, et al. 2015. Fine-scale chromatin interaction maps reveal the cis-regulatory landscape of human lincRNA genes. Nat Methods 12: 71-78. doi:10.1038/nmeth.3205

Martin M. 2011. Cutadapt removes adapter sequences from high-throughput sequencing reads. EMBnet.journal 17: 10. doi:10.14806/ej.17.1.200

McMurchy AN, Stempor P, Gaarenstroom T, Wysolmerski B, Dong Y, Aussianikava D, Appert A, Huang N, Kolasinska-Zwierz P, Sapetschnig A, et al. 2017. A team of heterochromatin factors collaborates with small RNA pathways to combat repetitive elements and germline stress. eLife 6: e21666. doi:10.7554/eLife.21666

Mifsud B, Tavares-Cadete F, Young AN, Sugar R, Schoenfelder S, Ferreira L Wingett SW, Andrews S, Grey W, Ewels PA, et al. 2015. Mapping longrange promoter contacts in human cells with high-resolution capture Hi-C. Nat Genet 47: 598-606. doi:10.1038/ng.3286

Nora EP, Lajoie BR, Schulz EG, Giorgetti L, Okamoto I, Servant N, Piolot T, van Berkum NL, Meisig J, Sedat J, et al. 2012. Spatial partitioning of the regulatory landscape of the X-inactivation centre. Nature 485: 381-385. doi:10.1038/nature 11049

Rao SS, Huntley MH, Durand NC, Stamenova EK, Bochkov ID, Robinson JT, Sanborn AL, Machol I, Omer AD, Lander ES, et al. 2014. A 3D map of the human genome at kilobase resolution reveals principles of chromatin looping. Cell 159: 1665-1680. doi:10.1016/j.cell.2014.11.021

Rao SSP, Huang SC, Glenn St Hilaire B, Engreitz JM, Perez EM, Kieffer-Kwon KR, Sanborn AL, Johnstone SE, Bascom GD, Bochkov ID, et al. 2017 Cohesin loss eliminates all loop domains. Cell 171: 305-320.e24. doi:10.1016/j.cell.2017.09.026

Robinson JT, Thorvaldsdóttir H, Winckler W, Guttman M, Lander ES, Getz G, Mesirov JP. 2011. Integrative genomics viewer. Nat Biotechnol 29: 2426. doi:10.1038/nbt.1754

Rowley MJ, Nichols MH, Lyu X, Ando-Kuri M, Rivera ISM, Hermetz K, Wang P, Ruan Y, Corces VG. 2017. Evolutionarily conserved principles predict 3D chromatin organization. Mol Cell 67: 837-852.e7. doi:10.1016/j .molcel.2017.07.022

Sati S, Cavalli G. 2017. Chromosome conformation capture technologies and their impact in understanding genome function. Chromosoma 126: 33-44 doi:10.1007/s00412-016-0593-6

Sexton T, Yaffe E, Kenigsberg E, Bantignies F, Leblanc B, Hoichman M, Parrinello H, Tanay A, Cavalli G. 2012. Three-dimensional folding and functional organization principles of the Drosophila genome. Cell 148: 458-472. doi:10.1016/j.cell.2012.01.010

Towbin BD, González-Aguilera C, Sack R, Gaidatzis D, Kalck V, Meister P, Askjaer P, Gasser SM. 2012. Step-wise methylation of histone H3K9 positions heterochromatin at the nuclear periphery. Cell 150: 934-947. doi:10.1016/j.cell.2012.06.051

Tsompana M, Buck MJ. 2014. Chromatin accessibility: a window into the genome. Epigenetics Chromatin 7: 33. doi:10.1186/1756-8935-7-33

Ursu O, Boley N, Taranova M, Wang YXR, Yardimci GG, Stafford Noble W, Kundaje A. 2018. GenomeDISCO: a concordance score for chromosome conformation capture experiments using random walks on contact map graphs. Bioinformatics 34: 2701-2707. doi:10.1093/bioinformatics/ bty 164

Wiesenfahrt T, Berg JY, Osborne Nishimura E, Robinson AG, Goszczynski B, Lieb JD, McGhee JD. 2016. The function and regulation of the GATA factor ELT-2 in the C. elegans endoderm. Development 143: 483-491. doi:10 $.1242 /$ dev.130914

Wingett S, Ewels P, Furlan-Magaril M, Nagano T, Schoenfelder S, Fraser P, Andrews S. 2015. HiCUP: pipeline for mapping and processing Hi-C data. F1000Res 4: 1310. doi:10.12688/f1000research.7334.1

Yang J, Fong HT, Xie Z, Tan JW, Inoue T. 2015. Direct and positive regulation of Caenorhabditis elegans bed-3 by PRDM1/BLIMP1 ortholog BLMP-1. Biochim Biophys Acta 1849: 1229-1236. doi:10.1016/j .bbagrm.2015.07.012

Received April 21, 2021; accepted in revised form December 19, 2021.

\section{Genome Research}

www.genome.org 


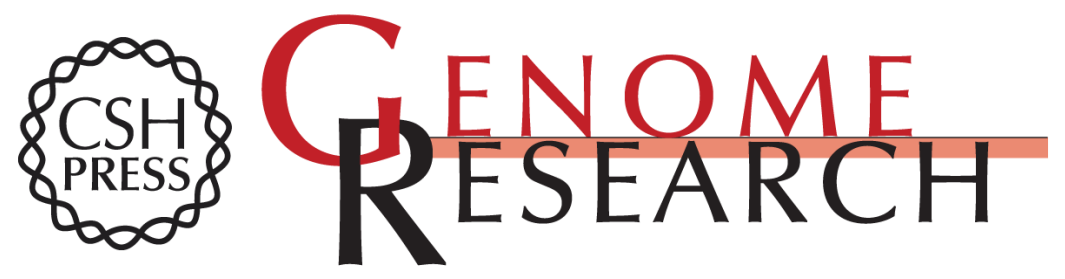

\section{Accessible Region Conformation Capture (ARC-C) gives high-resolution insights into genome architecture and regulation}

Ni Huang, Wei Qiang Seow, Alex Appert, et al.

Genome Res. 2022 32: 357-366 originally published online December 21, 2021

Access the most recent version at doi:10.1101/gr.275669.121

Supplemental Material

References

Open Access

Creative Commons

License

Email Alerting Service
http://genome.cshlp.org/content/suppl/2022/01/17/gr.275669.121.DC1

This article cites 55 articles, 12 of which can be accessed free at: http://genome.cshlp.org/content/32/2/357.full.html\#ref-list-1

Freely available online through the Genome Research Open Access option.

This article, published in Genome Research, is available under a Creative Commons License (Attribution 4.0 International), as described at http://creativecommons.org/licenses/by/4.0/.

Receive free email alerts when new articles cite this article - sign up in the box at the top right corner of the article or click here.

\section{Affordable, Accurate Sequencing.}

